\title{
Veils and Sensors: An artistic intervention with archival moving image material
}

\author{
Amanda Egbe \\ University of Bedfordshire \\ Luton, LU1 3JU, UK \\ amanda.egbe@beds.ac.uk
}

\section{INTRODUCTION}

This demonstration showcases experiments, and interventions with moving image archival materials by the author. The outcomes reflect a wider research into duplication practices in digital moving image archival practices. Artistic interventions are utilised to explore the technological and cultural gestures of these practices. The demonstrations are in the form of moving images artworks employing standard projection and mixed reality.

\section{FRAMING INTERVENTIONS IN MOVING IMAGE ARCHIVAL PRACTICES}

The context and framework for these artistic interventions stem from research into media technologies of reproduction. The focus on duplication offers the opportunity to explore the material trace of gestures in moving image materials through an interplay of artistic strategies and technological devices. Such gestures are expressed for example in the reproduction practices of Renaissance perspectival painting, where veils were utilised as a tool for the projection of objects onto a surface to trace an outline to paint with perspective. The technique not only produced perspective machines, which were made by artist engineers such as Durer, but it also created the culture of the Renaissance workshop where artists and technicians were trained in the techniques necessary to create perspective paintings such as frescos.

Similarly, the development of duplication technologies in moving image production and archiving has produced new techniques and tools. The enhancement of sensor-based scanning has for example allowed the analysis of moving image materials and their reproduction with more precision and less invasive handling of the materials. It has been necessary for the archivist as a curator engineer to extend their techniques within the digital realm. It is these techniques that the author's experiments visualise.

In both examples of the veil and the scanner the illusion of depth, colour and motion are areas of technique that are exemplified and developed as disciplining practices for the operator.

The work of artist filmmaker Ken Jacobs in his early works such as Tom Tom the Pipers' Son (1969) draws attention to the mode of technology in the performance of duplication through projection technologies. It also draws attention to the gestures of content creation within the image itself. Artists interventions can make visible this collaboration of mode and method to highlight the themes, motifs and gestures of artistic works as well as the technologies that are employed to produce them.

In his later work Jacob looks additionally to how the illusion of depth and motion is characterised by utilising 3D image making technology. Employing this effect he reworked TomTom the Piper's Son into an anaglyph, ANALGLYPH TOM (2008). The artist's strategies of a structural interrogation of the material allows for a tracing of artistic gestures, that is how notions of movement, and perception are characterised in the image's content and are then rendered as a visualisation, thus interpreting the image and producing a new work simultaneously, a digital revelatory practice (Turvey 2011).

\section{MOVING IMAGE EXPERIMENTS}

It is this framework of artistic intervention, alongside media and art historical inquiry, that is, developed further throughout these experiments. The works produced use materials from the DIASTOR scanner evaluation research project (Fleckiger, B. et al. 2018). The project aimed to assess the ability of moving image scanners for archival practices. A focus for the project was the 


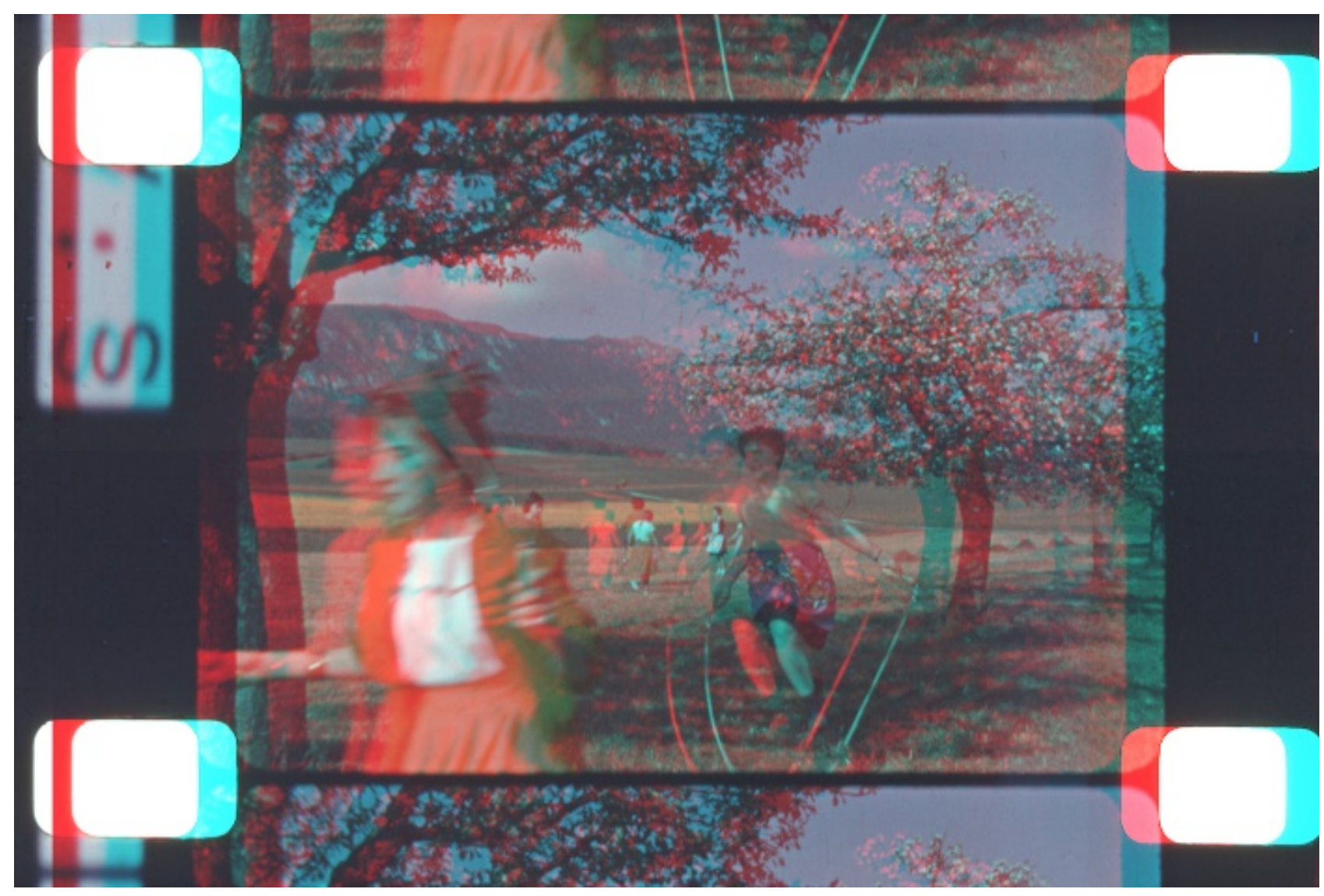

Figure 1: Author's anaglyph using an Ektachrome $35 \mathrm{~mm}$ film still.

effectiveness of scanners for specialist films whose colour profiles were hard for standard machines to replicate as they fell outside the profile of massproduced film materials. These included Technicolor, Ektachrome types of archival film materials.

The author's experiments utilise the software TouchDesigner and Adobe Premiere. A revelatory practice is created through the manipulation of visual information from the still image files and scanned sequences into looped moving works. The new works simultaneously refer to the profiles of the digital files, the illusion of movement in moving image practices, and a concern for depth with mixed and virtual environments. The aim of these experiments is to produce an artistic system that articulates and makes visible traces of technological and artistic gestures in moving image archival materials.

\section{REFERENCES}

Calter, P. (2008) Squaring the Circle: Geometry in art and architecture. John Wiley \& Sons.

Flueckiger, B., Pfluger, D., Trumpy, G., Croci, S., Aydın, T. and Smolic, A. (2018) Investigation of Film Material-Scanner Interaction, University of Zurich, Zurich Open Repository and Archive.

Jacobs, K. (1969) Tom Tom the Piper's Son, $16 \mathrm{~mm}$, color and b/w, silent, $115 \mathrm{~min}$.

Jacobs, K. (2008) ANAGLYPH TOM (Tom With Puffy Cheeks), anaglyph 3-D colour, sound, 109 $\min$.

Turvey, M. (2011) Ken Jacobs: Digital Revelationist. October, 137, pp.107-124. 